**TITLE**

ASP Conference Series, Vol. **VOLUME ${ }^{* * *}$, **YEAR OF PUBLICATION**

**NAMES OF EDITORS **

\title{
The relation between galactic properties and cluster structure
}

Bianca M. Poggianti

Osservatorio Astronomico di Padova-INAF, Italy

Terry J. Bridges

Anglo-Australian Observatory, Australia

M. Yagi

National Astronomical Observatory, Mitaka, Tokyo, Japan

Y. Komiyama

Department of Astronomy, University of Tokyo, Japan

Dave Carter

Liverpool John Moores University, Birkenhead, Wirral, UK

Bahram Mobasher

Space Telescope Science Institute, Baltimore, USA

S. Okamura

Department of Astronomy, University of Tokyo, Japan

N. Kashikawa

National Astronomical Observatory, Mitaka, Tokyo, Japan

\begin{abstract}
A satisfactory understanding of the origin of the dependence of galaxy properties on their environment has remained, so far, out of reach. In the light of numerous observational results and substantial theoretical progress obtained for clusters of galaxies in the last years, a primary goal is to understand how the star formation activity depends on cluster substructure, i.e. on the merging/accretion history of a cluster. In this contribution we present a case in which it is possible to identify the cluster environment, and in particular the intracluster medium and the recent infall history of galaxies onto the cluster, as the cause for an abrupt change in the star formation histories of a subset of galaxies in the Coma cluster.
\end{abstract}




\section{Introduction}

Fundamental galaxy properties such as star formation history, morphology and gas content vary in a systematic way from dense environments like clusters of galaxies to low density regions in the general field. Similarly, it has long been known that there are systematic variations of galaxy properties with cluster properties.

Already in 1974, on a sample of 15 clusters, Oemler tentatively divided clusters into three types. He noted that clusters with $\mathrm{cD}$ galaxies and rich in ellipticals have a smooth spherical distribution and are centrally concentrated, while clusters that are dominated by S0s are not as smooth, dense or centrally concentrated as cD clusters, and clusters of irregular appearance are dominated by spiral, star-forming galaxies. A few years later, in 1977, Bahcall first showed that the fraction of spiral galaxies in a cluster decreases rapidly with increasing X-ray luminosity (see also Edge \& Stewart 1991).

Subsequent studies on the correlation between galaxy properties and global properties of clusters have been innumerable, especially searching for correlations between the star formation activity in galaxies and the cluster X-ray luminosity (Smail et al. 1998, Ellingson et al. 2001, Balogh et al. 2002a), the cluster richness (Margoniner et al. 2001) and the presence of substructure (Wang \& Ulmer 1997, Metevier et al. 2000, Pimbblet et al. 2002). At some level the global cluster properties (X-ray and optical luminosities, richness, concentration etc.) are all correlated with each other, though with a large scatter, and it is hard to pin down what exactly is the primary correlation with galaxy properties that drives all the others.

A central question is surely how the star formation activity depends on cluster substructure, i.e. on the merging/accretion history of the cluster. There are some very good examples of cluster-cluster mergers where the star formation activity in galaxies, as measured by radio continuum emission, is exceptionally high, such as Abell 2125 (Dwarakanath \& Owen 1999) and Abell 2255 (Miller \& Owen 2003). In principle it is not justified to inequivocally attribute this activity to an "enhancement" of star formation due to the cluster-cluster merging: the activity observed could simply reflect an higher average activity in the substructures that are present in the cluster, given that the average star formation is known to be higher in lower density/less massive environment. Thus, the cause of the observed activity needs to be investigated on a one-by-one case. In Abell 2255, for example, the alignment of the star-forming galaxies along a preferential direction, perpendicular to the merger axis, strongly supports the hypothesis that the bursts of star formation were indeed triggered by the clustercluster merger, induced by ram pressure variations at the shock front (Miller \& Owen 2003). On the other hand, there are also merging clusters where only a low star formation activity is detected, suggesting that the gas reservoir in the galaxy populations of the two merging clusters must also play a role (Miller 2003). The dynamical state of the cluster and the type of galaxies that happen to be falling in at the time we observe it are the two crucial factors also for understanding the gas (HI) content of galaxies in clusters (van Gorkom 2003).

Galaxy properties are also known to be closely linked with their local environment, regardless of the "global" environment. The most striking evidence for this is the morphology-density relation (Dressler 1980, Dressler et al. 1997), 
paralleled by a star-formation/density relation (Lewis et al. 2002, Gomez et al. 2003). The higher incidence of ellipticals in regions of high local density, and the corresponding preference for spirals to reside in low density regions is known to be valid across a wide range of "global" environments, for both concentrated and irregular clusters, for rich and poor systems (Dressler 1980, Postman \& Geller 1984), though an additional second-order effect related to the global environment seems to be present, in the sense that at a given local density more massive and relaxed clusters show a small excess of early-type galaxies compared to less massive and irregular clusters (Dressler 1980, Balogh et al. 2002b). One of the open questions is therefore to what extent are the differences in galaxy properties in various types of clusters due to the fact that the average local environment varies as a function of the cluster type.

The findings cited above, together with many others in the literature, prove that both local and global effects are at work. In this talk we present the case of a cluster-related phenomenon, where it is possible to identify the cluster environment, and in particular the intracluster medium and the recent infall history of galaxies onto the cluster, as the cause for an abrupt change in the star formation histories of a subset of the Coma galaxies.

\section{Substructure and post-starburst galaxies in the Coma cluster}

In a recent spectroscopic survey of galaxies in the Coma cluster (Mobasher et al. 2001), we found that a significant fraction of the cluster dwarf galaxy population has post-starburst/post-starforming spectra (Poggianti et al. 2003, hereafter $\mathrm{P} 03$ ). This type of spectrum ("k+a", or " $\mathrm{E}+\mathrm{A}$ ") has no emission-line detected and equivalent width $\mathrm{EW}(\mathrm{H} \delta)>3 \AA$, indicating a galaxy with no current star formation activity which was forming stars at a vigorous rate in the recent past (last 1.5 Gyr).

Numerous spectroscopic surveys of galaxies in distant clusters have found significant populations of luminous $\mathrm{k}+\mathrm{a}$ galaxies (see for example Couch \& Sharples 1987, Abraham et al. 1996, Dressler \& Gunn 1992, Fisher et al. 1998, Dressler et al. 1999, Tran et al. 2003, but see also Balogh et al. 1999). However, $\mathrm{k}+\mathrm{a}$ galaxies as luminous as those in distant clusters $\left(M_{V} \leq-20\right)$ are absent in Coma, where $\mathrm{k}+\mathrm{a}$ 's are detected at magnitudes typically fainter than $M_{V} \sim-18$ (P03). The different luminosity distributions of $\mathrm{k}+\mathrm{a}$ galaxies in clusters at $z \sim 0.5$ and in Coma most likely reflects an evolution in the properties of the infalling galaxies, and provides further evidence of a "downsizing effect" (P03, see also Tran et al. 2003): the maximum luminosity/mass of galaxies with significant star formation activity seems to decrease at lower redshifts. In Coma we are observing late-type starforming galaxies becoming dwarf spheroidals (P03), while the descendants of $\mathrm{k}+\mathrm{a}$ 's at high redshift will be among the most massive early-type galaxies today (Tran et al. 2003).

In the color-magnitude diagram, a group of blue and a group of red $k+a$ 's can be easily distinguished in Coma. The average $\mathrm{EW}(\mathrm{H} \delta)$ of the blue group is significantly stronger than that of the red group. The blue, strong $\mathrm{k}+\mathrm{a}$ 's most likely correspond to "young" $\mathrm{k}+\mathrm{a}$ 's (observed soon after the termination of star formation, < $300 \mathrm{Myr}$ ) and the red, weaker k+a's are "old" ones (observed at a later stage of the evolution, 0.5-1.5 Gyr). Most of the blue $\mathrm{k}+\mathrm{a}$ 's, and a few of 
the red $\mathrm{k}+\mathrm{a}$ 's, have EWs stronger than $5 \AA$, testifying that a starburst occurred in the galaxy before star formation was quenched. Note that Balmer-enhanced galaxies in Coma, their luminosities and positions within the cluster have been the subject of several works by Caldwell, Rose and collaborators (e.g. Caldwell et al. 1993, see P03 for all references).

A suggestive clue about the possible physical mechanism responsible for the $\mathrm{k}+\mathrm{a}$ spectra comes from the recent X-ray mosaic observations of Coma obtained with XMM-Newton. Coma has two central dominant galaxies, NGC 4874 (a cD galaxy) and NGC4889 (a very bright elliptical), and another cD galaxy, NGC4839, that dominates a substructure South-West of the center (Fig. 1). Neumann et al. (2003) have recently identified and discussed X-ray substructure by fitting a smooth profile and subtracting it from the data. The residuals reveal several structures, that are shown as contours in Fig. 1: besides the well known NGC4839 South-West group, Neumann et al. identify a large residual to the West of the cluster centre ("Western structure" in Fig. 1) elongated along the North-South direction, and a filament-like structure South-East of the centre ("Eastern structure" in Fig. 1), elongated along the East-West direction. The temperature map shown in color in Fig. 1 sheds further light on the accretion history of Coma. Neumann et al. conclude that the region of high temperature observed between the Western structure and the Coma center is caused by the infall of this structure, either via compression or via shock waves. These authors consider the two maxima in the western structure to be likely the result of the disruption of a galaxy group during its infall, instead of two galaxy groups falling at the same time. In contrast, the South-Eastern structure is cooler than the mean cluster temperature and is associated with a low-mass galaxy group dominated by two large galaxies, NGC4911 and NGC4921. Based on the filamentary form of this structure, the same authors conclude it is observed during the infall process while being affected by ram pressure stripping close to the cluster centre.

The coincidence of the position of the strongest $\mathrm{k}+\mathrm{a}$ galaxies and the X-ray structures is striking. Four k+a's with $\operatorname{EW}(\mathrm{H} \delta)>5 \AA$ (green dots in Fig. 1) trace the edge of the Western structure towards the Coma centre. Another three are associated with the Eastern structure, all at its western boundary. Thus, young post-starbursts are distributed close to the edge of infalling substructures. In the case of the Western substructure this edge is the infalling front, while for the Eastern substructure it is unclear whether the group is moving to the West, as suggested by the appearance of the X-ray residuals, or to the East, as suggested by the positions of NGC4911 and NGC4921 (Neumann et al. 2003).

Overall, this strongly suggests that the $\mathrm{k}+\mathrm{a}$ spectra, i.e. the truncation of the star formation activity in these galaxies and possibly the previous starburst, could be the result of an interaction with the hot intracluster medium (ICM). Hence, as far as Coma $\mathrm{k}+\mathrm{a}$ galaxies are concerned, there is suggestive evidence that the origin of the $\mathrm{k}+\mathrm{a}$ spectrum is a cluster-related and, in particular, an ICM-related phenomenon that is closely connected with the dynamical state of the cluster. We note that the $\mathrm{H} \delta$ strength of the blue $\mathrm{k}+\mathrm{a}$ galaxies implies that star formation was truncated in these galaxies on a short timescale, i.e. short compared to the $\mathrm{k}+\mathrm{a}$ timescale of 1-1.5 Gyr. In fact, a slowly declining star formation activity such as that envisaged if galaxies simply lost their gas halo 
reservoir when becoming part of a group ("strangulation", e.g. Bower \& Balogh 2003) is not able to produce such strong Balmer lines.

It is worth stressing the importance of isolating the youngest $\mathrm{k}+\mathrm{a}$ galaxies using their blue colors and strong equivalent widths. The red $\mathrm{k}+\mathrm{a}$ phase has a timescale that is comparable to the core crossing time in a cluster like Coma, and any signature of the link between the truncation of star formation and the location within a substructure is thus erased in the older $\mathrm{k}+\mathrm{a}$ 's, while it is still detectable in the youngest subsample of $\mathrm{k}+\mathrm{a}$ 's.

It is also instructive to note that looking for a spatial segregation in the location of galaxies on the sky (Fig. 2) would not allow to establish a correlation between the star formation history of the $\mathrm{k}+\mathrm{a}$ galaxies and the substructure. The link with the dynamical history of Coma appears evident only once a detailed $\mathrm{X}$-ray map reveals the complicated structure in the hot intracluster gas.

The blue $\mathrm{k}+\mathrm{a}$ 's do show, however, a radial velocity distribution that is significantly different from that of the red $k+a$ 's and the global Coma dwarf population, as shown in Fig. 3. Their mean radial velocity is $8120 \pm 709 \mathrm{~km} \mathrm{~s}^{-1}$, with all but one at $v>7200 \mathrm{~km} \mathrm{~s}^{-1}$. In contrast, both the red $\mathrm{k}+\mathrm{a}$ 's and all faint galaxies with passive spectra have much lower mean velocities: $6992 \pm 761$ and $6854 \pm 244 \mathrm{~km} \mathrm{~s}^{-1}$, respectively. The blue $\mathrm{k}+\mathrm{a}$ galaxies have a relatively high velocity dispersion $\left(1250 \mathrm{~km} \mathrm{~s}^{-1}\right)$ already indicating that they cannot be part of a single infalling bound galaxy group of relatively small mass compared to the whole Coma cluster.

Interestingly, also the (few) emission line galaxies in the central region of Coma seem to trace the substructure: they are mostly found along the edge of the Western structure (Fig. 4), with the exception of \#15480 that is located just South of the Eastern structure. Emission-line galaxies are known to be proportionally more numerous in the South-West region than towards the central region of the cluster (Fig. 4).

\section{Conclusions}

The position of faint post-starburst galaxies in Coma relative to X-ray substructure in the cluster strongly suggests that the interruption of the star formation activity in these galaxies is a cluster-related phenomenon, most likely due to the impact with the intracluster medium. Post-starburst galaxies in Coma are much fainter than those observed in distant clusters, a difference probably reflecting a change in the galaxy populations infalling in clusters.

The dynamical state of the cluster and its recent history of accretion are clearly important factors in determining the galaxy properties observed. Identifying substructure, however, it is often hard, and no easily measurable "global" cluster property (such as for example X-ray luminosity or richness) is sufficient to characterize the cluster dynamical status. We have shown here an example

in which only a detailed analysis of the X-ray residuals has revealed the link between substructure and rapid changes in the star formation histories of some of the cluster galaxies.

Acknowledgments. BMP thanks the IAU and the organizers of this Symposium for their kind invitation and for generously supporting her participation with a IAU travel grant. 


\section{References}

Abraham, R.G., Smecker-Hane, T.A., Hutchings, J.B., Carlberg, R.G., Yee, H.K.C., Ellingson, E., Morris, S., Oke, J.B., Rigler, M., 1996, ApJ, 471, 694

Bahcall, N.,A., 1977, ApJ, 218, L93

Balogh, M. L., Morris, S. L., Yee, H. K. C., Carlberg, R. G., \& Ellingson, E., 1999, ApJ, 527, 54

Balogh, M.L., Bower, R.G., Smail, I., Ziegler, B.L., Davies, R.L., Gaztelu, A., Fritz, A., 2002a, MNRAS, 337, 256

Balogh, M.L., Smail, I., Bower, R.G., Ziegler, B.L., Smith, G.P., Davies, R.L., Gaztelu, A., Kneib, J.-P., Ebeling, H., 2002b, ApJ, 566, 123

Bower, R.G. \& Balogh, M.L., 2003 in Carnegie Observatories Astrophysics Series, Vol.3: Clusters of Galaxies: Probes of Cosmological Structure and Galaxy Evolution, eds. J.S. Mulchaey, A. Dressler and A. Oemler (Cambridge: Cambridge University Press), http://www.ociw.edu/ociw/symposia/series/symposium3/pro

Caldwell, N., Rose, J.A., Sharples, R.M., Ellis, R.S., Bower, R.G., 1993, AnJ, 106,473

Couch, W.J., Sharples, R.M., 1987, MNRAS, 229, 423

Dressler, A., 1980, ApJ, 236, 351

Dressler, A., Gunn, J.E., 1992, ApJS, 78, 1

Dressler, A., Oemler, A., Jr., Couch, W. J., Smail, I., Ellis, R. S., Barger, A., Butcher, H., Poggianti, B. M., Sharples, Ray M., 1997, ApJ, 490, 577

Dressler, A., Smail, I., Poggianti, B. M., Butcher, H., Couch, W. J., Ellis, R. S., \& Oemler, A. 1999, ApJS, 122, 51

Dwarakanath, K.S., Owen, F.N., 1999, AJ, 118, 625

Edge, A.C., Stewart, G.C., 1991, MNRAS, 252, 428

Ellingson, E., Lin, H., Yee, H. K. C., \& Carlberg, R. G. 2001, ApJ, 547, 609

Fisher, D., Fabricant, D., Franx, M., van Dokkum, P., 1998, ApJ, 498, 195

Gomez, P.L., Nichol, R.C., Miller, C.J., et al., 2003, ApJ, 584, 210

Lewis, I., Balogh, M., De Propris, R. et al., 2002, MNRAS, 334, 673

Metevier, A.J., Romer, A.K., Ulmer, M. P. 2000, AJ, 119, 1090

Miller, N.A., 2003, in Carnegie Observatories Astrophysics Series, vol.3: Clusters of Galaxies: Probes of Cosmological Structure and Galaxy Evolution (eds. J.S. Mulchaey, A. Dressler, and A. Oemler (Pasadena: Carnegie Observatories, http://www.ociw.edu/ociw/symposia/series/symposium3/proceedings.html)

Miller, N.A, Owen, F.N., 2003, AJ, 125, 2427

Mobasher, B., Bridges, T.J., Carter, D., Poggianti, B. M., et al., 2001. ApJS, 137,279

Neumann, D.M., Lumb, D.H., Pratt, G.W., Briel, U.G., 2003, A\&A, 400, 811

Oemler, A., 1974, ApJ, 194, 1

Pimbblet, K.A., Smail, I., Kodama, T., Couch, W., Edge, A.C., Zabludoff, A.I., O'Hely, E., 2002, MNRAS, 331, 333 
Poggianti, B.M., Bridges, T.J., Komiyama, Y., Yagi, M., Carter, D., Mobasher, B., Okamura, S., Kashikawa, N., 2003, ApJ in press (P03, astro-ph 0309449)

Postman, M., Geller, M.J., 1984, ApJ, 281, 95

Smail, I., Edge, A.C., Ellis, R. S., Blandford, R.D., 1998, MNRAS, 293, 124

Tran, K.-V.H., Franx, M., Illingworth, G., Kelson, D.D., van Dokkum, P., 2003, ApJ in press (astro-ph 0309460)

Treu, T., Ellis, R.S., Kneib, J.-P., Dressler, A., Smail, I., Czoske, O., Oemler, A., Natarajan, P., 2003, ApJ, 591, 53

van Gorkom, J., 2003, in Carnegie Observatories Astrophysics Series, vol.3: Clusters of Galaxies: Probes of Cosmological Structure and Galaxy Evolution (eds. J.S. Mulchaey, A. Dressler, and A. Oemler (Pasadena: Carnegie Observatories, http://www.ociw.edu/ociw/symposia/series/symposium3/proceedings.html)

Wang, Q.D., Ulmer, M.P., 1997, MNRAS, 292, 920 


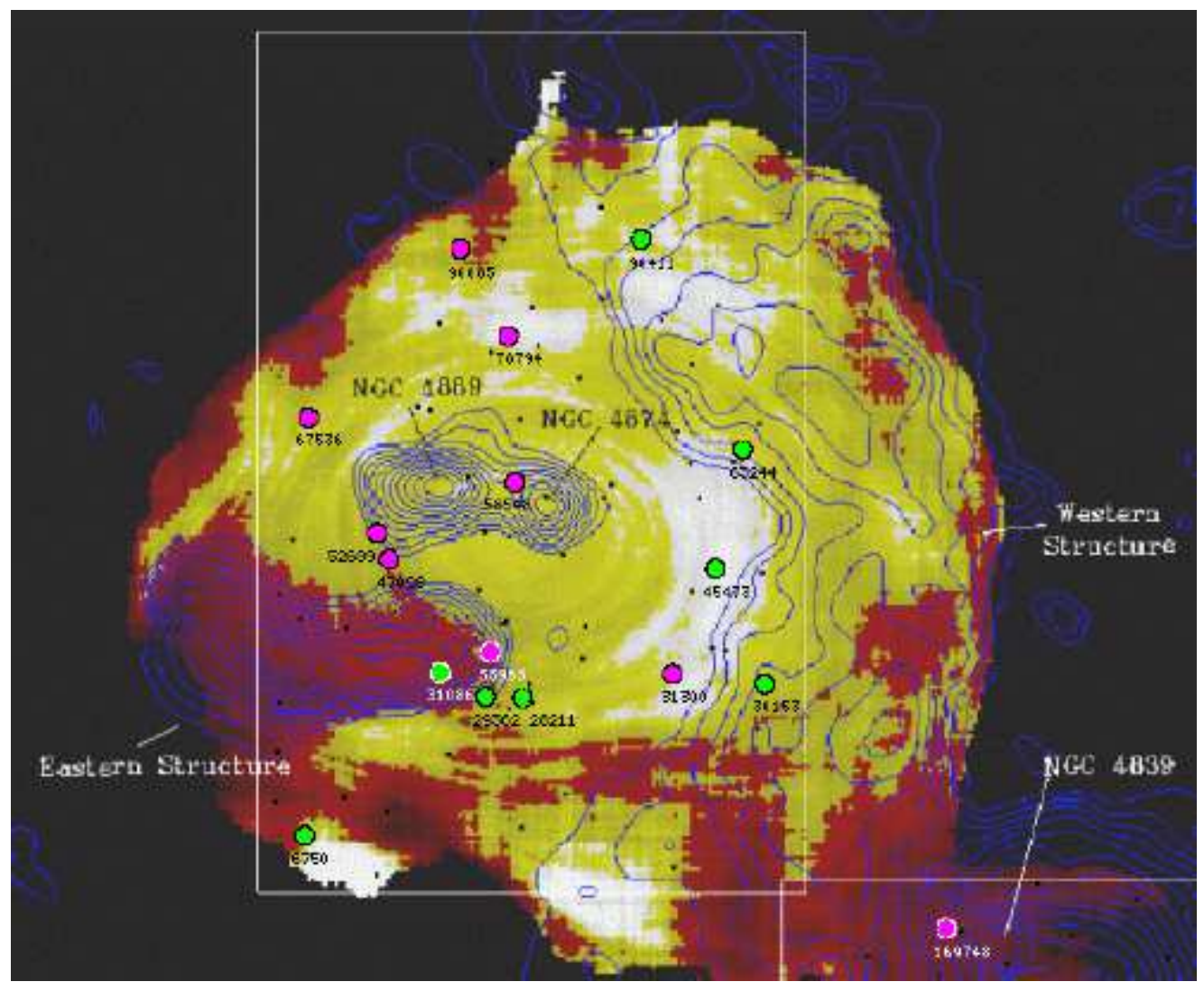

Figure 1. N.B. This plot is best viewed in color. Position of $\mathrm{k}+\mathrm{a}$ galaxies with respect to X-ray substructure and X-ray temperature map. Only the central field of Coma is shown here, see P03 for a full map. Strong-lined k+a's with $\mathrm{EW}(\mathrm{H} \delta)>5 \AA$ are shown as green dots, while weaker $\mathrm{k}+\mathrm{a}$ 's are plotted as magenta dots. Tiny black dots are dwarf Coma members with velocities $>7200 \mathrm{~km} \mathrm{~s}^{-1}$. X-ray residuals from Neumann et al. (2003) are plotted as contours and clearly identify two substructures (Western and Eastern substructures), in addition to the NGC4839 peak in the South-West and the excess of emission towards the two central galaxies (NGC4874 and NGC4889). The lowest contour and the step width between two contours are each 5 $\sigma$. The hardness ratio image (2-5 keV/0.5-2keV, Neumann et al. 2003) is shown in color. Red regions correspond to temperatures below 8 $\mathrm{keV}$, yellow to $k T>8 \mathrm{keV}$ and white regions to $k T>10 \mathrm{keV}$. The rectangles show the limits of the two fields of our photometric and spectroscopic survey (Coma1 towards the cluster center and Coma3 in the South-West). Each rectangle is about 1 by $1.5 \mathrm{Mpc}$. 


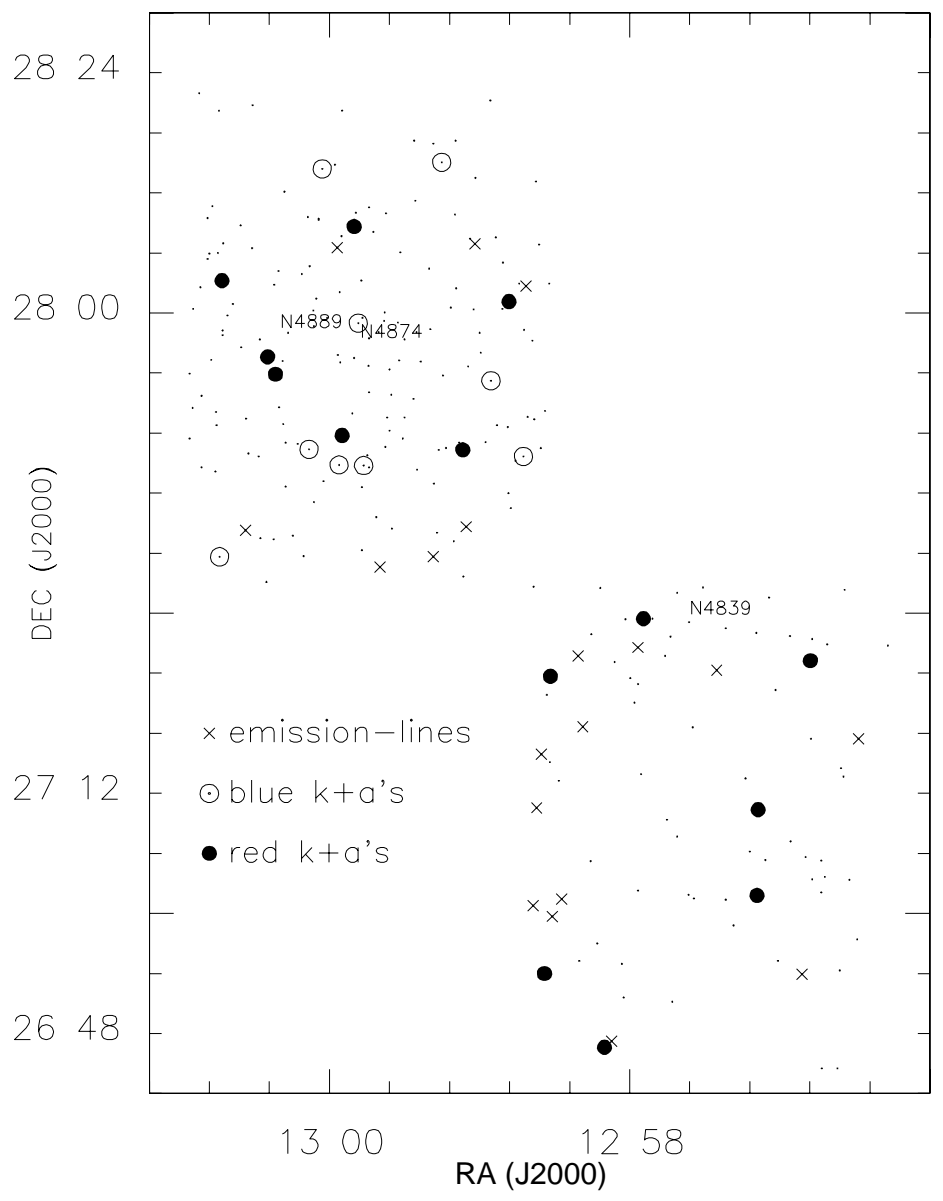

Figure 2. Projected position on the sky of galaxies with passive spectra (small dots), red and blue $\mathrm{k}+\mathrm{a}$ (large circles) and emission-line (crosses) galaxies. The location of the three dominant galaxies (N4874, N4889 and N4839) is labelled. 


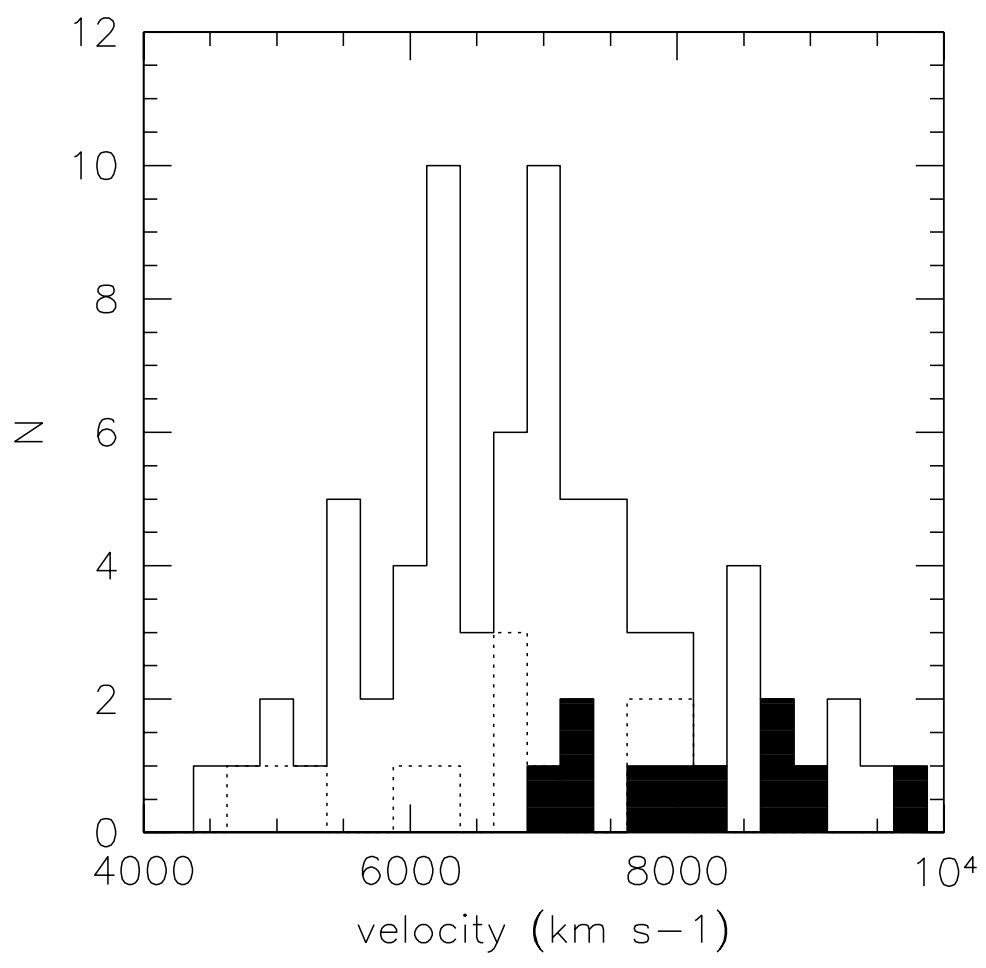

Figure 3. Velocity histograms of all Coma dwarfs (solid line), strong blue k+a's (filled histogram) and weak red k+a's (dashed line). 


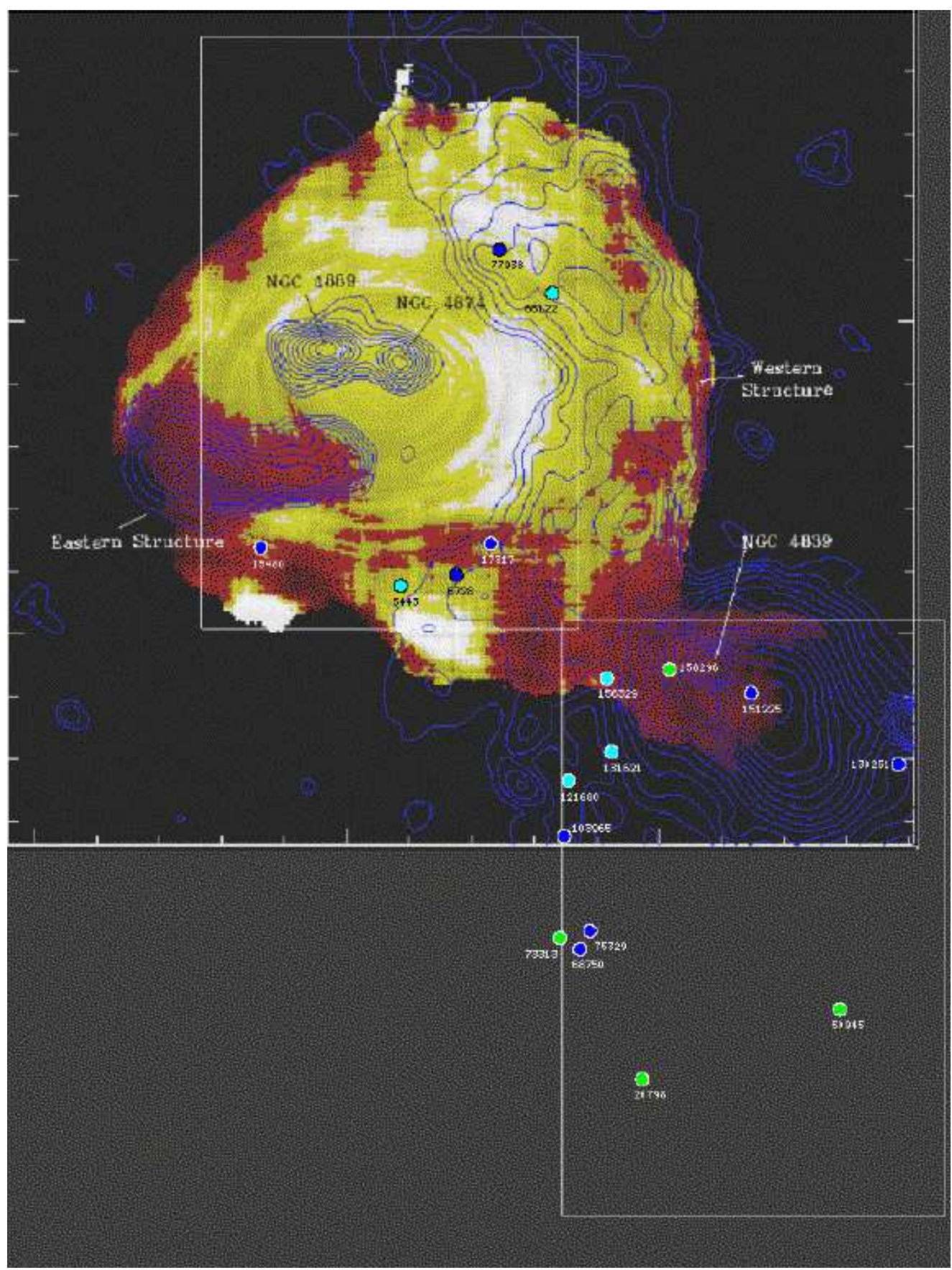

Figure 4. Same as Fig. 1, but now showing the positions of emissionline galaxies instead of $\mathrm{k}+\mathrm{a}$ 's. Dots of different colors represent galaxies with different levels of star formation activity, but all dots are associated with galaxies with ongoing star formation. 